hospital births. Following national consultation, in March 2014, the UK National Screening Committee made the decision to pilot the use of POS.

Aims

1. To assess adherence to the local guideline on pulse oximetry screening in neonates

2. To study the outcome of children with positive pulse oximetry test

Methodology A retrospective cross-sectional study of babies born in the Rosie Hospital was performed and samples were collected randomly. Data were collected from the hand-held postnatal care records.

Results POS results were documented in 552 of the 595 case notes studied. $55 \%$ of these had screening within recommended time (within $4-12 \mathrm{~h}$ of delivery). The majority of those performed outside of this time frame were after $12 \mathrm{~h}$.

16 of the 552 neonates had low $\mathrm{SpO}_{2}$ upon first measurement. Repeat pulse oximetry was documented in 13 of these 16 neonates; values were $\geq 95 \%$ in 11 infants on repeat. One infant was found to have complete atrio-ventricular septal defect (AVSD) whilst one had persistent fetal circulation.

The 3 remaining neonates with low $\mathrm{SpO}_{2}$ had no documented follow up or outcome in the maternal hand-held notes prior to discharge. Further investigation of hospital notes confirmed one of these infants had significant sepsis and mild persistent pulmonary hypertension of newborn (PPHN), one was treated for suspected sepsis, whilst the third had a structurally normal heart with transitional circulation.

Conclusions Overall, new clinical practice in POS has been widely embraced by staff in Cambridge; however, adherence to the guideline and documentation could be improved in the Rosie Hospital. In our study, false positives accounted for $<0.8 \%$ of results; most infants with false positive POS had other noncardiac pathologies.

\section{G161 TRANSPOSITION OF GREAT ARTERIES, A 15 YEAR EXPERIENCE OF 74 PATIENTS IN WALES: INCORPORATION OF OUTFLOW TRACT VIEW IN THE ANTENATAL SCANS LEADING TO BETTER ANTENATAL DETECTION AND CLINICAL OUTCOMES}

${ }^{1} S$ Nittur, ${ }^{2} \mathrm{~A}$ Wong, ${ }^{3} \mathrm{M}$ Shethalli, ${ }^{2} \mathrm{O}$ Uzun. ${ }^{1}$ Paediatrics, Royal Glamorgan Hospital, Llantrisant, UK; ${ }^{2}$ Paediatric Cardiology, University Hospital of Wales, Cardiff, UK; ${ }^{3}$ Paediatric Intensive Care, University Hospital of Wales, Cardiff, UK

\subsection{6/archdischild-2015-308599.157}

Background Antenatal detection rate of transposition of the great arteries (TGA) has remained very low around 25\% in the UK. This may have improved with the addition of outflow tract view to the routine 20 week foetal anomaly screening protocols with better overall outcomes.

Patients and methods All children who were diagnosed with simple TGA at our centre for Paediatric Cardiology over a 15 year period were included in this study. Clinical case notes were retrospectively reviewed and outcome data was evaluated.

Results There were 74 patients; 54 diagnosed postnatally, 20 antenatally. All 8 preterm deliveries were in postnatally diagnosed group. 32 had simple TGA and 42 had additional defects. Male: female ratio was 3.5:1. Antenatal detection rate improved from $0-20 \%$ to $75 \%$ in the recent years. $40 \%$ of cases diagnosed postnatally were unwell at presentation and most of them needed ventilatory support. Majority of the patients in our cohort needed prostin infusion following delivery and 60\% of them underwent balloon septostomy before arterial switch operation was performed. Only one out of 74 patients had Mustard's operation in our series. Postoperative complications were more common in postnatally diagnosed patients (26\% vs $10 \%)$. There were no deaths in the antenatally detected group. Early mortality rate was $6.8 \%$ and operative mortality was $1.4 \%$ with no postoperative deaths being recorded after 2007. Echo abnormalities were seen in $83 \%$ at 2 years and $91 \%$ at 10 years follow up with neo aortic regurgitation being the commonest. Intervention free survival was $96 \%$. $7 \%$ were on medications mainly due to impaired LV function. Growth and development issues, exercise intolerance and arrhythmias were uncommon in our series. Survival rate at 5 years was $88.1 \%$.

Conclusion Inclusion of outflow tract view has led to substantial improvement in antenatal detection of TGA. This, in turn, has made a major impact on the clinical outcomes owing to better cardiovascular status at presentation, lower postoperative complications and reduced mortality rates.

\section{G162 IMPACT OF NATIONAL PRENATAL SCREENING GUIDELINES ON THE DETECTION RATES OF TRANSPOSITION OF THE GREAT ARTERIES IN NEONATES UNDERGOING THE ARTERIAL SWITCH PROCEDURE}

DC Gardner, JL Heaps, CB Jones, JSL Lim. Cardiology Department, Alder Hey Children's Hospital, Liverpool, UK

\subsection{6/archdischild-2015-308599.158}

Aims Prenatal diagnosis of transposition of the great arteries (TGA) has been shown to improve pre-operative clinical condition and long term outcome. In 2008 the National Institute of Clinical Excellence (NICE) published guidelines advising that ventricular outflow tracts should be assessed as part of the routine prenatal assessment and this was consolidated in the 2010 FASP guidelines.

This had a large impact on sonographer training requirements and although skills are increasing, prenatal detection differs significantly by region. Our aim was to review the effect of these new guidelines on our prenatal detection rate and evaluate the impact of these changes on our patient population.

Method All patients undergoing the arterial switch procedure (ASO) for simple TGA between 2001 and 2013 were identified. This enabled assessment of patient outcomes before and after the introduction of the 2008 NICE guidance. Patients with septal defects were included but those with additional CHD were excluded.

A retrospective review of patient records was performed. Data was gathered regarding patient demographics, timing of diagnosis and subsequent admission to the cardiac centre. For patients admitted, their pre-operative status, surgical intervention and immediate and long term outcomes were reviewed.

Results 219 patients with simple TGA +/- septal defects who had the arterial switch procedure were identified during the 13 year period. The rate of antenatal diagnosis has been consistently increasing since 2008 (see Figure 1), from 11\% prior to 2008 to $41 \%$ from 2008 onwards and 62\% in the most recent year.

Conclusions Nationally antenatal diagnosis of congenital heart disease is improving according to CCAD data. Locally our prenatal diagnosis rate has been consistently increasing since 2007 but there remains room for improvement with higher prenatal detection rates in other regions. Further analysis is required to determine the outcome following a prenatal diagnosis both regionally and nationally. 